\title{
Letter
}

\section{On Kasner solution in Bianchi I $f(T)$ cosmology}

\author{
Maria A. Skugoreva ${ }^{1, a}$, Alexey V. Toporensky ${ }^{1,2, b}$ \\ ${ }^{1}$ Kazan Federal University, Kremlevskaya 18, Kazan 420008, Russia \\ 2 Sternberg Astronomical Institute, Lomonosov Moscow State University, Moscow 119991, Russia
}

Received: 6 February 2018 / Accepted: 30 April 2018 / Published online: 12 May 2018

(c) The Author(s) 2018

\begin{abstract}
Recently the cosmological dynamics of an anisotropic Universe in $f(T)$ gravity became an area of intense investigations. Some earlier papers devoted to this issue contain contradictory claims about the nature and propertied of vacuum solutions in this theory. The goal of the present paper is to clarify this situation. We compare properties of $f(T)$ and $f(R)$ vacuum solutions and outline differences between them. The Kasner solution appears to be an exact solution for the $T=0$ branch, and an asymptotic solution for the $T \neq 0$ branch. It is shown that the Kasner solution is a past attractor if $T<0$, being a past and future attractor for the $T>0$ branch.
\end{abstract}

The Kasner solution, being one of the first known exact solutions in relativistic cosmology [1] continues to be one of the most important exact solutions in general relativity (GR) or its modifications. One of the reasons is that despite this being a vacuum solution, it is a good approximation near a cosmological singularity for almost all matter sources (except for a stiff fluid) in a flat anisotropic Universe. Moreover, a general cosmological singularity is believed to be constructed as an infinite series of consecutive epochs, each of them being a particular Kasner solution with a good accuracy (though a mathematical proof of this scenario is still absent in full details; see, for example, [2]) — the famous BelinskiiKhalatnikov-Lifshitz (BKL) scenario [3]. Therefore the Kasner set of solutions provides "building blocks" for the BKL picture.

If we assume that GR needs some modifications at UV scale, it is natural to expect that such modifications should change the behavior near a cosmological singularity significantly. That is why the fate of the Kasner solution in modified gravity theories is an area of intense investigations. A lot of efforts have been devoted to Kasner solutions and their modifications in quadratic gravity. We remind the reader that the Kasner solution is a solution for an anisotropically expand-

\footnotetext{
a e-mail: masha-sk@mail.ru

b e-mail: atopor@rambler.ru
}

ing Universe with scale factors changing as powers of time. These power exponents are subject of two conditions giving us their sum as well as the sum of their squares (both sums are equal to unity). In quadratic gravity, two different situations were identified:

- If the equations of motion are of the second order, as in GR (that is, in Gauss-Bonnet gravity), the power-law solution for the scale factor is an asymptotic solution. In the high-curvature regime, these two conditions for power exponents are different from those in the GR Kasner solution [4-6], while the GR Kasner solution is an asymptotic solution in the low-curvature regime.

- In fourth order gravity (like $R+R^{2}$ or a general quadratic gravity) the Kasner solution (with the same conditions for the exponents) is an exact vacuum solution. However, since the phase space has two additional dimensions in comparison with GR, a Kasner solution in quadratic gravity may be in some situations unstable $[7,8]$.

Recently a new class of modified gravity theories has started to attract much attention. It is based on the Teleparallel Equivalent to General Relativity (TEGR) - a theory first considered by Einstein in the 1920s [9-11] where the LeviCivita connection (torsion-free, non-zero curvature) has been replaced by Weitzenböck connection [12] (curvature-free, non-zero torsion), and curvature scalar $R$ in the action by the torsion scalar $T$. It appears that despite different mathematical backgrounds, TEGR and GR are identical at the level of the equations of motion. For a review see, for example, the book [13]. Now it is well known that in cases more complicated than the standard Einstein-Hilbert action, the theory based on torsion has the equations of motion different from the theory based on curvature. In particular, $f(T)$ theory is not equivalent to $f(R)$ theory, if the function $f$ is not a linear function.

This motivated studies of cosmological dynamics in $f(T)$ gravity. Recently many papers on this topic have appeared, mostly concentrating on FRW cosmology (see, for example, 
[14-17] and the references therein. Anisotropic cosmology is the next natural step in this direction. However, $f(T)$ theory has its own problems connected with the lack of local Lorentz invariance $[18,19]$. This leads to a situation when not all tetrads corresponding to the chosen metric form gives us the correct equations of motion [20,21], so the separate problem of choosing a so-called "proper tetrad" [22] appears. Alternatively, proper non-zero spin connections must be associated with a given tetrad [23]. Careful investigation of this problem in connection with anisotropic metrics is still missing. In such a situation we can use the heuristic argument of [23] that if a tetrad (or spin connection) is chosen in a bad way, the resulting equations of motion should be (in some sense) pathological. The most common pathology is a requirement that the second derivative of $f$ with respect to $T$ vanishes, which evidently brings us back to TEGR. So, a reasonable current strategy may be in choosing the simplest tetrad associated with a Bianchi I metric, and in studying the corresponding equations of motion, if they do not show such pathologies.

This strategy has been implemented recently in several papers [24-27]. The resulting equations of motion appear to be nonpathological. These papers have, however, some contradictory statements. In particular, the study of Ref. [24] concludes that a vacuum solution exists only for a particular $f(T)=\sqrt{-T}$ theory and it must be isotropic. On the contrary, Ref. [27] claims that a Kasner solution is still a solution for $f(T)$ theory, though it is unstable. The fact that the Kasner solution remains as a solution can easily be checked by direct substitution to the equations of motion. Thus it seems that the second above-mentioned alternative could be realized. However, the problem is that once the tetrad is fixed, the number of degrees of freedom in $f(T)$ theory and in TEGR is the same. In $f(R)$ gravity the instability of the Kasner solution (which is the exact solution in that theory) is due to the extra degrees of freedom, which are absent in $f(T)$ theory in question. This contradiction needs a careful analysis, which is the goal of the present paper. We will see below that none of the two quadratic gravity alternatives regarding a Kasner solution can be true for $f(T)$ cosmology where we meet a third, different situation.

We consider cosmological models in modified teleparallel gravity $f(T)$, where (as in TEGR) the dynamical variables are tetrad fields $\mathbf{e}_{A}\left(x^{\mu}\right)$; here Greek indices are space-time and capital Latin indices relate to the tangent space-time. The metric tensor is given by $g_{\mu \nu}=\eta_{\mathrm{AB}} e_{\mu}^{A} e_{v}^{B}$, where $\eta_{\mathrm{AB}}=$ $\operatorname{diag}(1,-1,-1,-1)$.

The action of $f(T)$ theory without matter has the form

$S=\frac{1}{16 \pi G} \int e(f(T)) d^{4} x$,

where $e=\sqrt{-g}$ is the determinant of the tetrad, $f(T)$ is a general differentiable function of the torsion scalar $T$. Units $\hbar=c=1$ will be used.
We choose the following diagonal tetrad:

$e_{\mu}^{A}=\operatorname{diag}(1, a(t), b(t), c(t))$,

which relates to the Bianchi I metric $\mathrm{d} s^{2}=\mathrm{d} t^{2}-a^{2}(t) \mathrm{d} x^{2}-$ $b^{2}(t) \mathrm{d} y^{2}-c^{2}(t) \mathrm{d} z^{2}$, where $a(t), b(t), c(t)$ are scale factors. The torsion scalar for the chosen tetrad (2) is

$T=-\frac{2}{a b c}(c \dot{a} \dot{b}+b \dot{a} \dot{c}+a \dot{b} \dot{c})$,

where a dot denotes the derivative with respect to time. We can rewrite the expression for $T$ (3) using anisotropic Hubble parameters $H_{a} \equiv \frac{\dot{a}}{a}, H_{b} \equiv \frac{\dot{b}}{b}, H_{c} \equiv \frac{\dot{c}}{c}$,

$T=-2\left(H_{a} H_{b}+H_{a} H_{c}+H_{b} H_{c}\right)$,

which reduces to $T=-6 H^{2}$ in the isotropic case $a(t)=$ $b(t)=c(t), H_{a}=H_{b}=H_{c}=H$.

Varying the action (1) with respect to the chosen tetrad (2) the equations of motion are obtained (see, for example [24]):

$$
\begin{aligned}
& -2 T f_{T}+f(T)=0 \\
& \dot{T} f_{T T}\left(H_{b}+H_{c}\right)+\frac{f}{2}+f_{T}\left(\dot{H}_{b}+\dot{H}_{c}+\left(H_{b}\right)^{2}+\left(H_{c}\right)^{2}\right. \\
& \left.\quad+2 H_{b} H_{c}+H_{a} H_{b}+H_{a} H_{c}\right)=0 \\
& \dot{T} f_{T T}\left(H_{a}+H_{c}\right)+\frac{f}{2}+f_{T}\left(\dot{H}_{a}+\dot{H}_{c}+\left(H_{a}\right)^{2}+\left(H_{c}\right)^{2}\right. \\
& \left.\quad+2 H_{a} H_{c}+H_{a} H_{b}+H_{b} H_{c}\right)=0 \\
& \dot{T} f_{T T}\left(H_{a}+H_{b}\right)+\frac{f}{2}+f_{T}\left(\dot{H}_{a}+\dot{H}_{b}+\left(H_{a}\right)^{2}+\left(H_{b}\right)^{2}\right. \\
& \left.\quad+2 H_{a} H_{b}+H_{a} H_{c}+H_{b} H_{c}\right)=0 .
\end{aligned}
$$

Here we denote $f_{T}=\frac{\mathrm{d} f(T)}{\mathrm{d} T}, f_{T T}=\frac{d^{2} f(T)}{\mathrm{d} T^{2}}$.

We investigate cosmological models with the Lagrangian density function $f(T)=T+f_{0} T^{N}$, where $f_{0}, N$ are parameters. It is important to notice that the constraint equation (5) in this model is an algebraic relation for the torsion scalar $T$. This means that, in contrast to $f(R)$ theory where there exists a differential equation for the curvature scalar (see, for example, [28]), and $R$ has a dynamics, the torsion scalar at the entire trajectory can be equal to only one value of a discrete set of possible values. In particular for the power-law model studied in the present paper, $T$ can belong to only two branches of solutions. Substituting $f(T)=T+f_{0} T^{N}$ and $f_{T}(T)=1+N f_{0} T^{N-1}$ into (5) we find

$T\left(f_{0}(1-2 N) T^{N-1}-1\right)=0$ 
then

$$
\begin{aligned}
& \text { 1). } T=0 \\
& \text { 2). } T^{N-1}=\frac{1}{f_{0}(1-2 N)}=\text { const, } N \neq \frac{1}{2} .
\end{aligned}
$$

(1) The case of $T=0$.

If $T=0$ then $f_{T}=1$ and the field equations (6)-(8) have the form

$$
\begin{aligned}
& \dot{H}_{b}+\dot{H}_{c}+\left(H_{b}\right)^{2}+\left(H_{c}\right)^{2}+2 H_{b} H_{c}+H_{a} H_{b}+H_{a} H_{c}=0, \\
& \dot{H}_{a}+\dot{H}_{c}+\left(H_{a}\right)^{2}+\left(H_{c}\right)^{2}+2 H_{a} H_{c}+H_{a} H_{b}+H_{b} H_{c}=0, \\
& \dot{H}_{a}+\dot{H}_{b}+\left(H_{a}\right)^{2}+\left(H_{b}\right)^{2}+2 H_{a} H_{b}+H_{a} H_{c}+H_{b} H_{c}=0 .
\end{aligned}
$$

This system of the field equations coincides with that in GR for the Bianchi I metric. Therefore, the Kasner solution [1] $a(t)=a_{0} t^{p_{1}}, b(t)=b_{0} t^{p_{2}}, c(t)=c_{0} t^{p_{3}}$ with $p_{1}+p_{2}+$ $p_{3}=1,\left(p_{1}\right)^{2}+\left(p_{2}\right)^{2}+\left(p_{2}\right)^{2}=1$ is the exact solution for this system. Moreover, as in GR it is a general vacuum solution for the Bianchi I metric.

It is easy to see that there is no de Sitter solution with $H_{a}=H_{b}=H_{c}=H_{d S}=$ const $\neq 0$ on this branch.

Since the constraint equation is an algebraic, but not a differential equation for $T$ it is impossible to vary $T$, setting it to some non-zero value on the branch in question. So, all corrections to GR vanish on this branch of Bianchi I vacuum solutions. This situation has no analogs in both $f(R)$ and Gauss-Bonnet gravity. Only if matter sources are taken into account, corrections to GR become non-vanishing (in this case $T$ evidently is not zero). That is why the question of stability of a vacuum Kasner solution on the $T=0$ branch is a meaningless question, because this branch contains no other vacuum solutions.

(2) The case of $T^{N-1}=\frac{1}{f_{0}(1-2 N)}=$ const.

For $T^{N-1}=\frac{1}{f_{0}(1-2 N)}=$ const we get $f_{T}=\frac{1-N}{1-2 N}$ and from (5) $f=2 T f_{T}=2 T \frac{1-N}{1-2 N}$. Then the equations of motions (6)-(8) reduce to

$$
\begin{aligned}
& \frac{1-N}{1-2 N}\left[T+\left(\dot{H}_{b}+\dot{H}_{c}+\left(H_{b}\right)^{2}+\left(H_{c}\right)^{2}+2 H_{b} H_{c}\right.\right. \\
& \left.\left.\quad+H_{a} H_{b}+H_{a} H_{c}\right)\right]=0, \\
& \frac{1-N}{1-2 N}\left[T+\left(\dot{H}_{a}+\dot{H}_{c}+\left(H_{a}\right)^{2}+\left(H_{c}\right)^{2}+2 H_{a} H_{c}\right.\right. \\
& \left.\left.\quad+H_{a} H_{b}+H_{b} H_{c}\right)\right]=0, \\
& \frac{1-N}{1-2 N}\left[T+\left(\dot{H}_{a}+\dot{H}_{b}+\left(H_{a}\right)^{2}+\left(H_{b}\right)^{2}+2 H_{a} H_{b}\right.\right. \\
& \left.\left.\quad+H_{a} H_{c}+H_{b} H_{c}\right)\right]=0 .
\end{aligned}
$$

In the limit $\left|H_{a}\right| \rightarrow \infty,\left|H_{b}\right| \rightarrow \infty,\left|H_{c}\right| \rightarrow \infty$, we can neglect the constant term $f / 2$ and find the asymptotic Kasner solution $a(t)=a_{0}\left|t-t_{0}\right|^{p_{1}}, b(t)=b_{0}\left|t-t_{0}\right|^{p_{2}}, c(t)=$ $c_{0}\left|t-t_{0}\right|^{p_{3}}, t \rightarrow t_{0}$ with $p_{1}+p_{2}+p_{3}=1,\left(p_{1}\right)^{2}+\left(p_{2}\right)^{2}+$ $\left(p_{2}\right)^{2}=1$.

Moreover, the exact de Sitter solution exists $H_{d S}=$ $\pm \sqrt{-T / 6}$ for the negative values of $T$. We write down conditions of the existence of a de Sitter solution [27]:

(a) if $N$ is even, then $f_{0}>0$,

(b) if $N$ is odd, then $f_{0}<0$.

We apply the numerical integration of the initial system (6)-(8) and check the constraint equation $f_{0}(1-2 N) T^{N-1}-$ $1=0$ at each step of the integration. It should be noted that we can use (10) to decrease the number of degrees of freedom. However, in GR numerics, especially on singular solutions, it is better to use the constraint equation for checking the numerical accuracy. This is a widespread practice. The numerical work is carried out for $N=2$ and $N=3$ with the initial values of Hubble parameters near the de Sitter point and we plot the evolution of trajectories going to the past, that is, from the de Sitter Universe to the Kasner one. This result is shown in Fig. 1. This figure appears to be the same as the corresponding figure of [27], so we disagree with this paper only on some points of interpretation, not in numerical results (see the summary below in our conclusions).

We also calculate the following dimensionless parameters:

$P_{1}(t)=-\frac{H_{a}^{2}}{\dot{H}_{a}}, \quad P_{2}(t)=-\frac{H_{b}^{2}}{\dot{H}_{b}}, \quad P_{3}(t)=-\frac{H_{c}^{2}}{\dot{H}_{c}}$,

which tend to Kasner indices $p_{1}, p_{2}, p_{3}$ when the cosmological evolution approaches the Kasner phase (see Figs. 2, 3, 4). We can see from these plots that soon before reaching a cosmological singularity, these parameters tend to some constant values, as they should for the Kasner solution. Note that since we trace the evolution backward in time starting from $t=0$, the cosmological singularity appears at some time $t_{0}$, which depends on the trajectory. The form of the Kasner solution above has been written for the general case (including $\left(t_{0}=0\right)$ and $\left.\left(t_{0} \neq 0\right)\right)$. Figure 2 represents the time behavior of $P_{1}$, the results for $P_{2}$ and $P_{3}$ are qualitatively the same. In Figs. 3 and 4 we plot time dependences of $P_{1}+P_{2}+P_{3}$ and $\left(P_{1}\right)^{2}+\left(P_{2}\right)^{2}+\left(P_{3}\right)^{2}$, showing that they tend to their Kasner values for all studied trajectories. This justifies our statement that a Kasner solution is an asymptotic solution for $T+f_{0} T^{N}$ cosmology near a cosmological singularity.

Finally, we consider the case of $T=$ const $>0$, where the de Sitter attractor does not exist. What happens here, can be seen from Fig. 5 where we plot the evolution of the volume of the Universe. In order to calculate the time dependences of the volume factor $V(t) \equiv a(t) b(t) c(t)$ we integrate the system (6)-(8) with an addition three differential equations for scale factors: $\dot{a}=H_{a} a, \dot{b}=H_{b} b, \dot{c}=H_{c} c$. It appears 


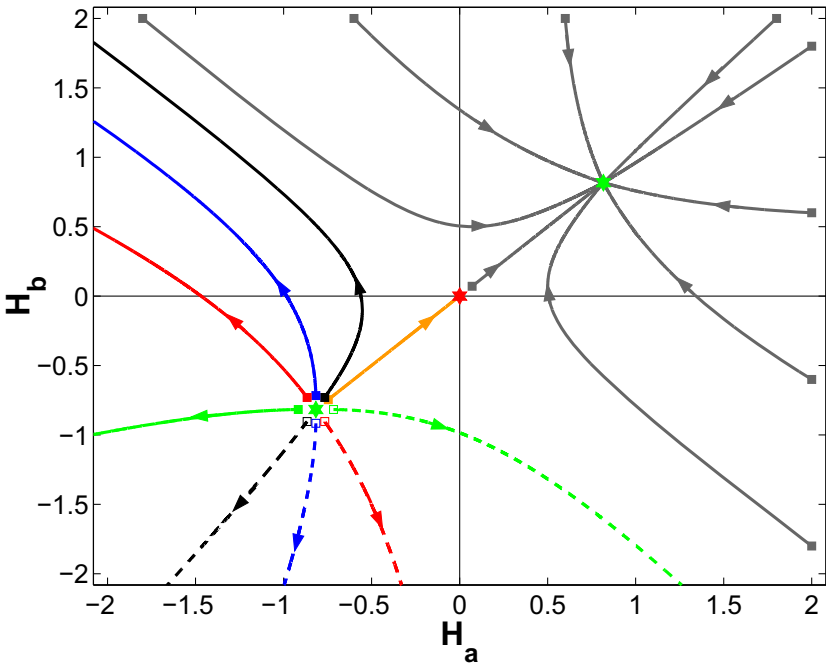

Fig. 1 Phase portraits for $N=2, f_{0}=\frac{1}{12}$ (left) and $N=3$, $f_{0}=-\frac{1}{5}$ (right). Red and green stars denote the point $(0 ; 0)$ and de Sitter ones $\left(H_{d S} ; H_{d S}\right)$ correspondingly. Initial points $\left(H_{a}(0) ; H_{b}(0)\right)$ have the following coordinates: 1 for black, blue, red and green squares $H_{a}(0)$ equaling values from $H_{d S}-0.1$ to $H_{d S}+0.1$, where $H_{d S}<0$, with step 0.05 and corresponding values $H_{b}(0)=$

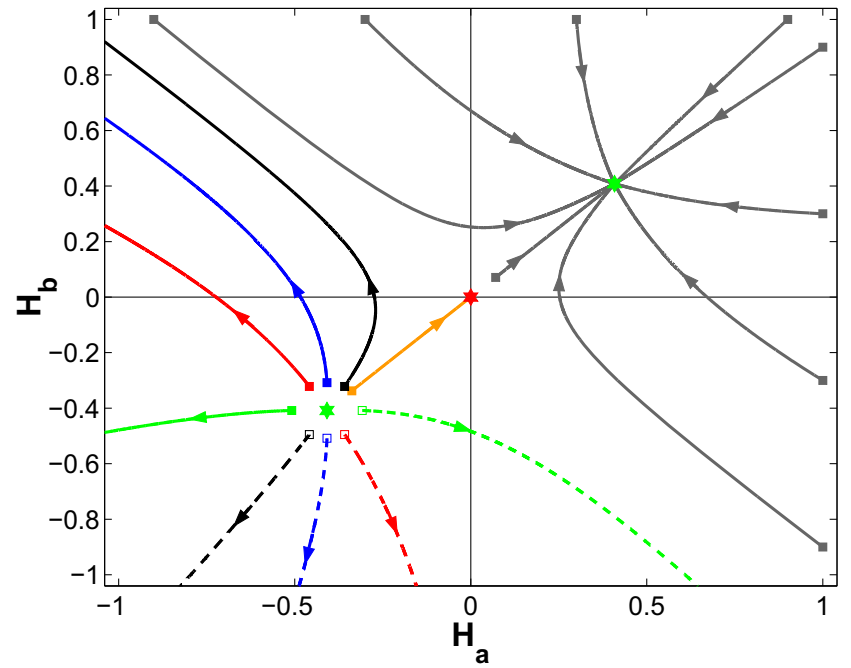

$\pm \sqrt{(0.1)^{2}-\left(H_{a}(0)-H_{d S}\right)^{2}}+H_{d S}, 2$ for the orange square $H_{a}(0)=$ $H_{b}(0)=H_{d S}+0.1 / \sqrt{2}$, where $H_{d S}<0.3$ in the left plot for gray squares. a $H_{a}(0)$ equaling values from -1.8 to 1.8 with step 1.2 and $H_{b}(0)=2$, b $H_{a}(0)=2$ and $H_{b}(0)$ equaling values from -1.8 to 1.8 with step $1.2,4$ in the right graph for gray squares a $H_{a}(0)$ equaling values from -0.9 to 0.9 with step 0.6 and $H_{b}(0)=1, \mathbf{b} H_{a}(0)=1$ and $H_{b}(0)$ equaling values from -0.9 to 0.9 with step 0.6

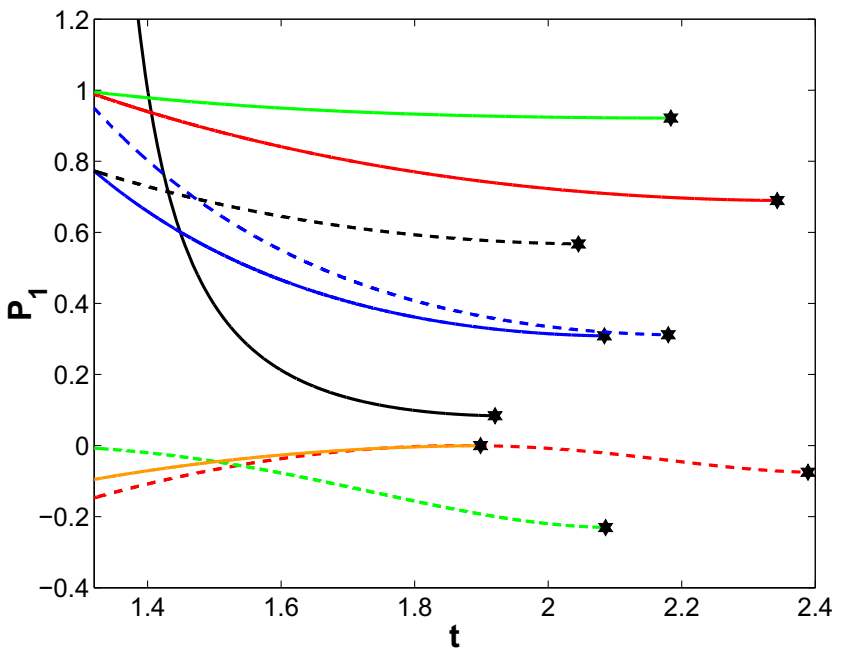

Fig. 2 The final stage of the evolution of the dimensionless parameter $P_{1}(t)$ for $N=2, f_{0}=\frac{1}{12}$ (left) and $N=3, f_{0}=-\frac{1}{5}$ (right). Initial values of $H_{a}(0), H_{b}(0)$ are chosen as in Fig. 1 (see the corresponding colors and types of line). The black star denotes the moment of a cosmological singularity

that the volume reaches its maximum value at some time of the cosmological evolution, and after that the Universe starts to recollapse. After that, the evolution ends in a Big Crunch singularity, where the volume shrinks to zero at some time $t_{0}$. As near the Big Crunch singularity the absolute values of Hubble parameters grow infinitely, we can again neglect the constant $T$ in the equations of motion, which means that the metric tends to the Kasner solution. In Figs. 6 and 7 we show numerically that for the branch $T=$ const $>0$ the cosmological evolution begins and ends in a Kasner solution as the sums $P_{1}+P_{2}+P_{3}$ and $\left(P_{1}\right)^{2}+\left(P_{2}\right)^{2}+\left(P_{3}\right)^{2}$ tend to unity at late time. The saddle point in Fig. 6 (left) indicates the Minkowski vacuum solution $H_{a}=H_{b}=H_{c}=0$, any trajectory initially approaches this point; then, after reaching the maximal expansion point, it departs from it. The initial Kasner indices differ from the final ones (see Fig. 6, right). We have checked that, while starting from arbitrary $H_{a}, H_{b}$ (and finding the initial $H_{c}$ from the constraint equation), we 

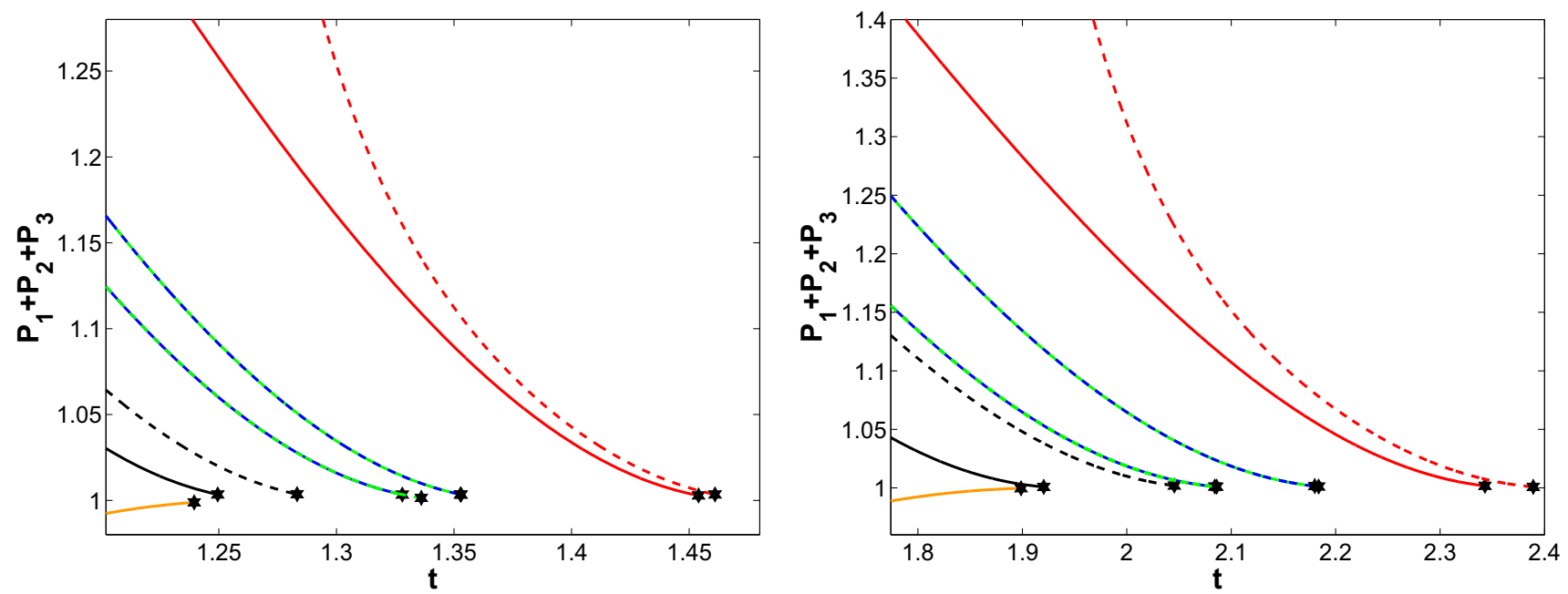

Fig. 3 The final stage of the evolution of the sum $P_{1}+P_{2}+P_{3}$ for $N=2, f_{0}=\frac{1}{12}$ (left) and $N=3, f_{0}=-\frac{1}{5}$ (right). Initial values of $H_{a}(0)$, $H_{b}(0)$ are chosen as in Fig. 1 (see the corresponding colors and types of line). The black star denotes the moment of a cosmological singularity
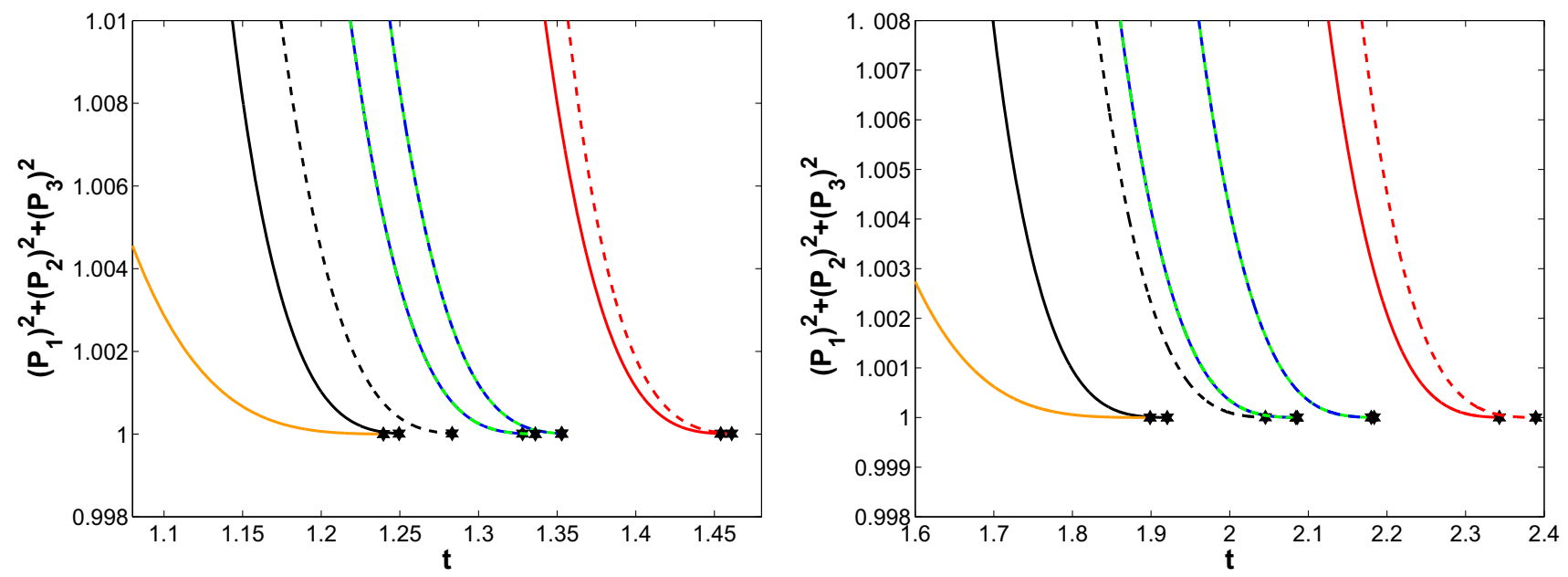

Fig. 4 The final part of the evolution of the sum $\left(P_{1}\right)^{2}+\left(P_{2}\right)^{2}+\left(P_{3}\right)^{2}$ for $N=2, f_{0}=\frac{1}{12}$ (left) and $N=3, f_{0}=-\frac{1}{5}$ (right). Initial values of $H_{a}(0), H_{b}(0)$ are chosen as in Fig. 1 (see the corresponding colors and types of line). The black star denotes the moment of a cosmological singularity

get the same evolution from the Kasner solution near the Big Bang at some time $t_{1}$; then, after recollapse, we have a Kasner solution near the Big Crunch at some time $t_{2}$. In the intermediate region the meaning of the parameters $P_{1}$, $P_{2}$ and $P_{3}$ is rather obscure, they can even diverge during cosmological evolution, since $\dot{H}_{i}$ entering the denominator in the definition of the corresponding $P_{i}$ may go through zero.

To conclude we would like to underline on which points we agree and disagree with Ref. [27]. Since there are some subtleties, it is better to distinguish between the cases when the Kasner solution is an exact solution and an asymptotic solution.

We agree with [27] that the Kasner solution can be an exact solution in $f(T)$ cosmology. However, it happens only on the
$T=0$ branch of vacuum solutions where the equations of motion coincide exactly with those for GR (any deviations from GR appears only when matter is taken into account). Therefore the question of the stability of the Kasner solution in this situation is meaningless - the Kasner solution is the general vacuum solution on the $T=0$ branch.

On the contrary, the Kasner solution is an asymptotic solution on the second branch. In this case it is possible to ask for its stability, and we agree with [27] that it is unstable, and the cosmological evolution is generally directed from the Kasner to the de Sitter solution. However, in this situation the Kasner solution cannot be an exact solution of $f(T)$ cosmology.

So, the statement that the Kasner solution satisfies the equations of motion in $f(T)$ theory made in the Introduction of [27], and the statement that it is unstable (which is the 


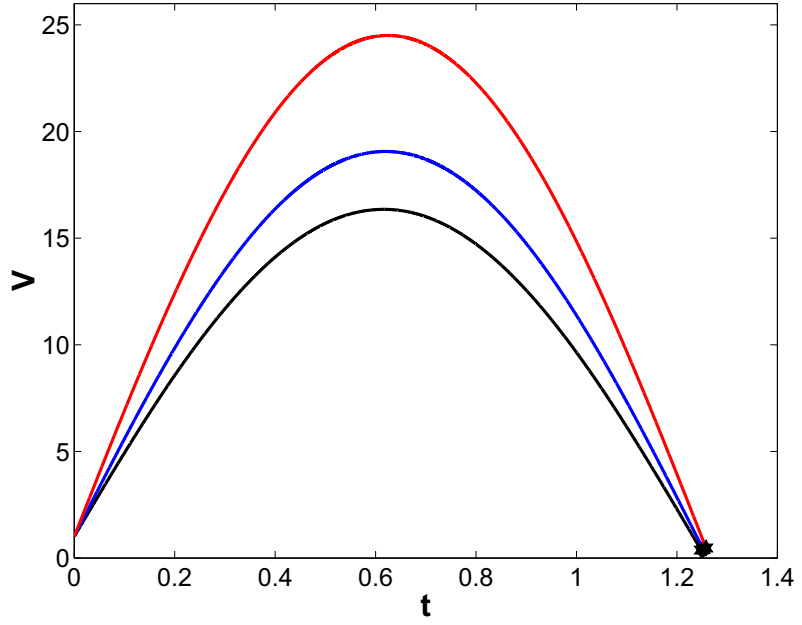

Fig. 5 The evolution of the volume factor $V(t)$ for $N=2, f_{0}=$ $-\frac{1}{12}$. Initial values of $H_{a}(0), H_{b}(0)$ are chosen as in Fig. 6 (see the corresponding colors of solid curves) and starting values of scale factors are $a(0)=1, b(0)=1, c(0)=1$. The black star denotes the moment of a cosmological singularity in the future

main result of [27]) are both correct only when applied to appropriate branches of solutions. They are not correct for another branch. However, in [27] these statements have been formulated in general, without any reference to particular branches.

Moreover, our study does not support the claim of [27] that the Kasner solution is a saddle. On the contrary, we can see from Figs. 1, 2, 3, and 4 that on the $T \neq 0$ branch, where

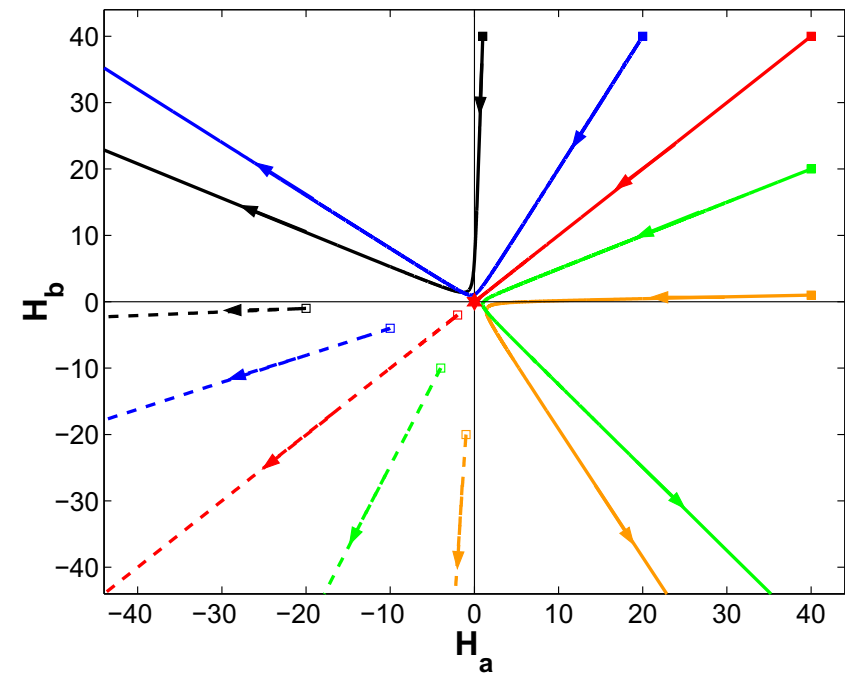

Fig. 6 The phase portrait (left) and the evolution of the dimensionless parameter $P_{1}(t)$ (right) for $N=2, f_{0}=-\frac{1}{12}$. Initial values of $H_{a}(0), H_{b}(0)$ are 1).-5). for solid curves and 6).-10). for dashed those: 1). $H_{a}(0)=1, H_{b}(0)=40$ (black), 2). $H_{a}(0)=20, H_{b}(0)=40$ (blue), 3). $H_{a}(0)=40, H_{b}(0)=40$ (red), 4). $H_{a}(0)=40, H_{b}(0)=$ $20($ green $), 5) . H_{a}(0)=40, H_{b}(0)=1$ (orange), 6). $H_{a}(0)=-20$, the Kasner solution is unstable, it represents a source in an expanding Universe. This means that it is stable into the past, and that it is an attractor for a contracting Universe.

Our results can be understood in another way if we note that the vacuum equations of motion of the models $f(T)=$ $T+f_{0} T^{N}$ for the branch $T=$ const $\neq 0$ coincide with those of anisotropic Bianchi I models of GR with cosmological constant $\Lambda$, where $T>0$ corresponds to $\Lambda<0(T<0$ corresponds to $\Lambda>0$ ). Therefore, a cosmological evolution is directed from a Kasner to a de Sitter solution in the case $T<0$, and from one Kasner solution to the point of maximal expansion (see Fig. 7) and back to another Kasner solution in the case of positive $T$.

As for the statement of [24] that a vacuum solution can exist only for $f(T)=\sqrt{-T}$ theory, to our mind, it follows from an incorrect interpretation of the constraint equation (5). This equation may be considered as a differential equation which should get us such $f(T)$ that the constraint is valid for all $T$. In this interpretation the authors of [24] are correct. However, this equation can be considered as an algebraic one which gives us some particular $T$ for which the constraint equation has solutions. This situation is known in $f(R)$ cosmology. The equation for a vacuum de Sitter solution $2 f(R)-R f^{\prime}(R)=0$ when considered as a differential equation gives a particular $f(R)=R^{2}$, for which a de Sitter solution with any $R$ exists. On the contrary, the same equation being an algebraic equation gives for another $f(R)$ some particular $R$ for which a de Sitter solution exists. In $f(R)$ theory this is restricted by de Sitter solutions only, because in

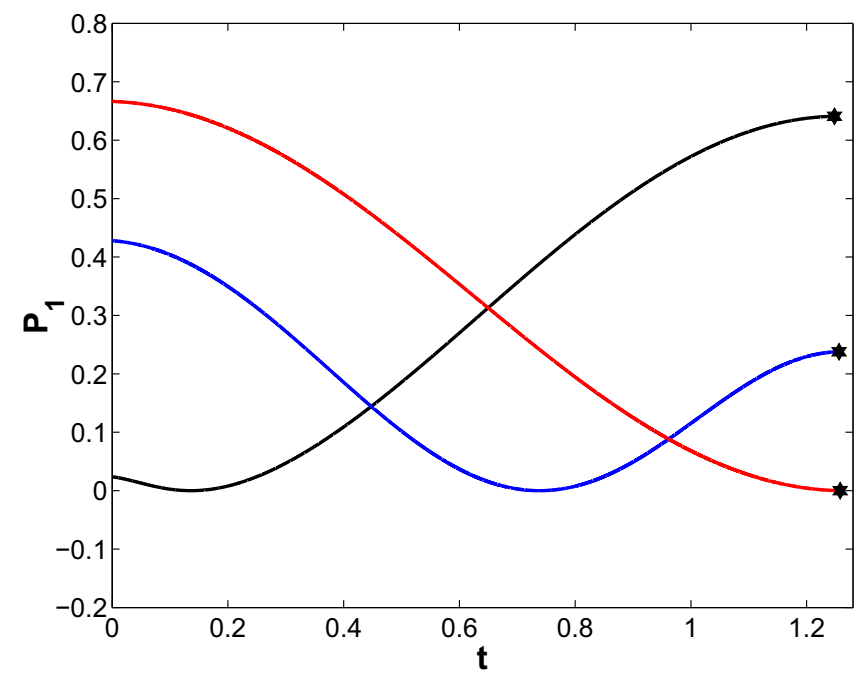

$H_{b}(0)=-1$ (black), 7). $H_{a}(0)=-10, H_{b}(0)=4$ (blue), 8). $H_{a}(0)=$ $-2, H_{b}(0)=-2$ (red), 9). $H_{a}(0)=-4, H_{b}(0)=10$ (green), 10). $H_{a}(0)=-1, H_{b}(0)=20$ (orange). The red star denotes the point $(0 ; 0)$ and the black star is the moment of a cosmological singularity in the future 

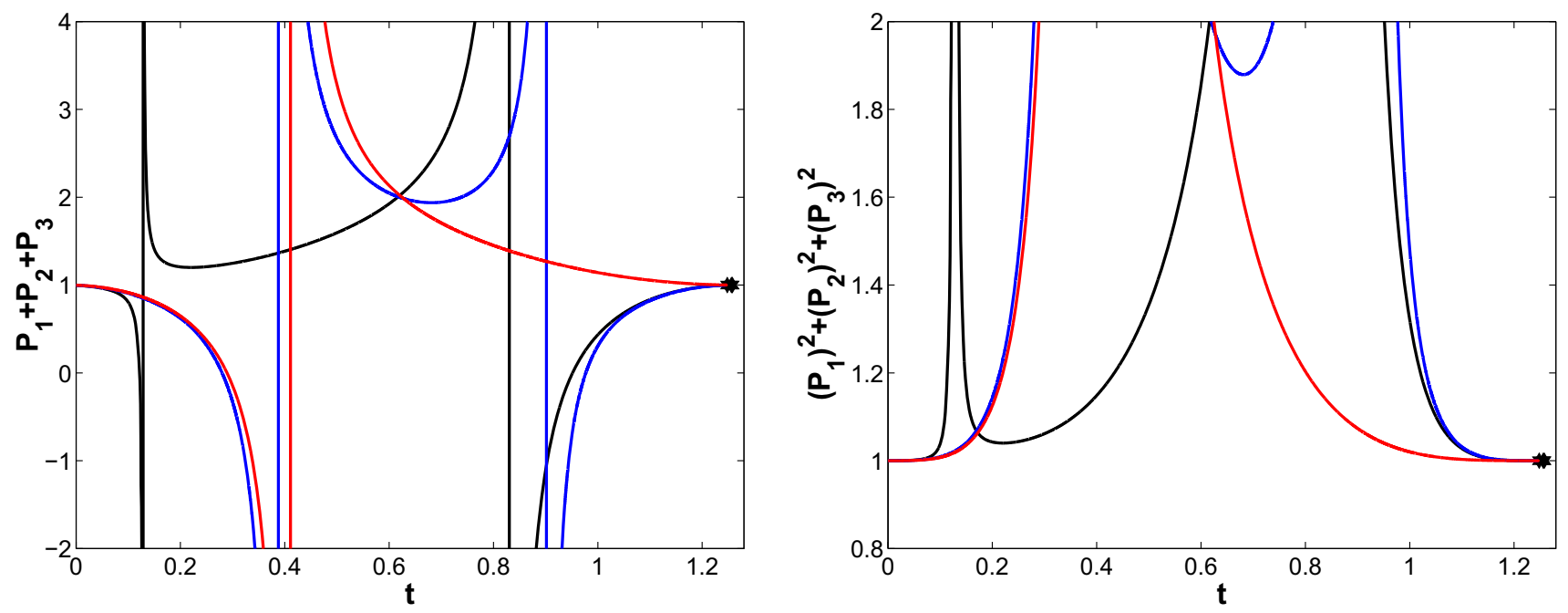

Fig. 7 The evolution of sums $P_{1}+P_{2}+P_{3}$ (left) and $\left(P_{1}\right)^{2}+\left(P_{2}\right)^{2}+\left(P_{3}\right)^{2}$ (right) for $N=2, f_{0}=-\frac{1}{12}$. Initial values of $H_{a}(0), H_{b}(0)$ are chosen as in Fig. 6 (see the corresponding colors of solid curves). The black star denotes the moment of a cosmological singularity in the future

a general situation there are other derivative terms. In $f(T)$ the situation is general. If we search for any vacuum solution, there is no need for this solution to be valid for any $T$, so the constraint equation should be considered as an algebraic one.

We remind the reader that all results of the present paper have been obtained under the suggestion that the tetrad $(1, a, b, c)$ is the proper tetrad for Bianchi I cosmology. Our results, showing that the Kasner solution is either the exact general solution or an asymptotic solution stable to the past, indicate that it is reasonable to search for an analog of BKL oscillations in anisotropic $f(T)$ cosmology with spatial curvature. However, this needs to identify proper tetrads for other Bianchi metrics (or, in another formulation of the theory, correct non-zero spin connections for these cases), which is a separate interesting and currently unsolved problem of $f(T)$ gravity.

Acknowledgements The work was supported by RSF Grant No. 1612-10401 and by the Russian Government Program of Competitive Growth of Kazan Federal University.

Open Access This article is distributed under the terms of the Creative Commons Attribution 4.0 International License (http://creativecomm ons.org/licenses/by/4.0/), which permits unrestricted use, distribution, and reproduction in any medium, provided you give appropriate credit to the original author(s) and the source, provide a link to the Creative Commons license, and indicate if changes were made. Funded by SCOAP ${ }^{3}$.

\section{References}

1. E. Kasner, Am. J. Math. 43, 217 (1921)

2. J.M. Heinzle, C. Uggla, Class. Quant. Grav. 26, 075015 (2009)

3. V.A. Belinskii, E.M. Lifshitz, I.M. Khalatnikov, Sov. Phys. Usp. 13, 745 (1971)
4. N. Deruelle, Nucl. Phys. B 327, 253 (1989)

5. N. Deruelle, L. Farina-Busto, Phys. Rev. D 41, 3696 (1990)

6. A. Toporensky, P. Tretyakov, Grav. Cosmol. 13, 207 (2007)

7. J.D. Barrow, S. Hervik, Phys. Rev. D 74, 124017 (2006)

8. A. Toporensky, D. Müller, Gen. Rel. Grav. 49(1), 8 (2017)

9. A. Einstein, Sitz. Preuss. Akad. Wiss., 217 (1928)

10. A. Einstein, ibid. 224 (1928)

11. A. Unzicker and T. Case, [arXiv:physics/0503046]

12. R. Weitzenböck, Invarianten Theorie (Noordhoff, Groningen, 1923)

13. R. Aldrovandi, J.G. Pereira, Teleparallel Gravity: An Introduction (Springer, Dordrecht, 2013)

14. R.C. Nunes, S. Pan, E.N. Saridakis, JCAP 08, 011 (2016)

15. R.C. Nunes, A. Bonilla, S. Pan, E.N. Saridakis, Eur. Phys. J. C 77, 230 (2017)

16. M. Hohmann, L. Järv, U. Ualikhanova, Phys. Rev. D 96, 043508 (2017)

17. A. Awad, W. El Hanafy, G.G.L. Nashed, E.N. Saridakis, JCAP 02, 052 (2018)

18. B. Li, T.P. Sotiriou, J.D. Barrow, Phys. Rev. D 83, 064035 (2011)

19. T.P. Sotiriou, B. Li, J.D. Barrow, Phys. Rev. D 83, 104030 (2011)

20. R. Ferraro, F. Fiorini, Phys. Rev. D 91, 064019 (2015)

21. C. Bejarano, R. Ferraro, M.J. Guzmán, Eur. Phys. J. C 77, 825 (2017)

22. R. Ferraro, F. Fiorini, Phys. Lett. B 702, 75 (2011)

23. M. Krśśak, E. Saridakis, Class. Quantum. Grav. 33, 115009 (2016)

24. M.E. Rodrigues, M.J.S. Houndjo, D. Saez-Gomez, F. Rahaman, Phys. Rev. D 86, 104059 (2012)

25. Y.-F. Cai, S. Capoziello, M. De Laurentis, E.N. Saridakis, Rept. Prog. Phys. 79(4), 106901 (2016)

26. A. Paliathanasis, J.D. Barrow, P.G.L. Leach, Phys. Rev. D 94, 023525 (2016)

27. A. Paliathanasis, J.L. Said, J.D. Barrow, Phys. Rev. D 97, 044008 (2018)

28. D. Müller, A. Ricciardone, A.A. Starobinsky, A. Toporensky, Eur. Phys. J. C 78, 311 (2018) 